Note

\section{Postprandial Existence Ratio between Taurine- and Glycine-conjugated Bile Acids in Rat Ileal Lumen}

\author{
Masaru Kitagawa, Kimikazu Iwami \\ and Fumio IBUKI \\ Department of Agricultural Chemistry, \\ Kyoto Prefectural University, Shimogamo, \\ Sakyo-ku, Kyoto 606, Japan
}

Received December 4, 1990

Intraluminal bile acids are intimately involved in fat digestion and absorption, which subsequently are for the most part taken up from the terminal ileum probably by a $\mathrm{Na}^{+}$-dependent active transport mechanism. ${ }^{1)}$ Taurineconjugated bile acids $(\mathrm{T})$, although being the predominant rat biliary bile acids, ${ }^{2)}$ are replaced by glycine-conjugated bile acids $(G)$ under such nutritional conditions as to exhaust the taurine pool. ${ }^{3)}$ Even though not to that extent, dietary factors affecting the availability of sulfurcontaining amino acids cause some changes in the existence ratio between taurine and glycine-conjugated bile acids (T/G ratio $){ }^{4)}$ In this preliminary report, rats meal-fed with alternation of non- and high-protein diets twice a day were examined for postprandial changes in the intraluminal $T / G$ ratio in the ileum.

Albino male Wistar rats weighing about $300 \mathrm{~g}$ were housed for 4 weeks in an air-conditioned room with a half-day light/dark cycle (lights on, 8:00-20:00) on a meal-feeding schedule with access to a $40 \%$ casein diet between 9:00 and 11:00 and to a non-protein diet between 20:00 and 22:00 (group A) or vice versa (group B). A third group of rats given a $20 \%$ casein diet during both prescribed time periods were regarded as the control (group C). At 11:00, 14:00 and 18:00 on the 29th day, the respective animals were sacrificed to excise the distal third of the small intestine, of which the intraluminal leavings and washings were combined (arbitrarily defined as the intraluminal content) and subjected to separation and quantification of the bile acids. In brief, an aliquot of the intraluminal content $(100 \mu \mathrm{l})$ was treated at $65^{\circ} \mathrm{C}$ for $15 \mathrm{~min}$ with $0.2 \mathrm{~N} \mathrm{NaOH}$, which was followed by thin-layer chromatography using $n$-butanol-acetic acid-water $(10$ : $1: 1, v / v)$ as the developing solvent. ${ }^{5}$ The bands corresponding to $T$ and $G$ on the silica gel plate were individually scraped off, and their steroid contents were determined by absorbance measurement at $375 \mathrm{~nm}$ after treatiung at $65^{\circ} \mathrm{C}$ for $60 \mathrm{~min}$ with $65 \% \mathrm{H}_{2} \mathrm{SO}_{4}{ }^{5}$ ) The amount of total bile acids in the intraluminal content was likewise determined after an ethanol extraction under

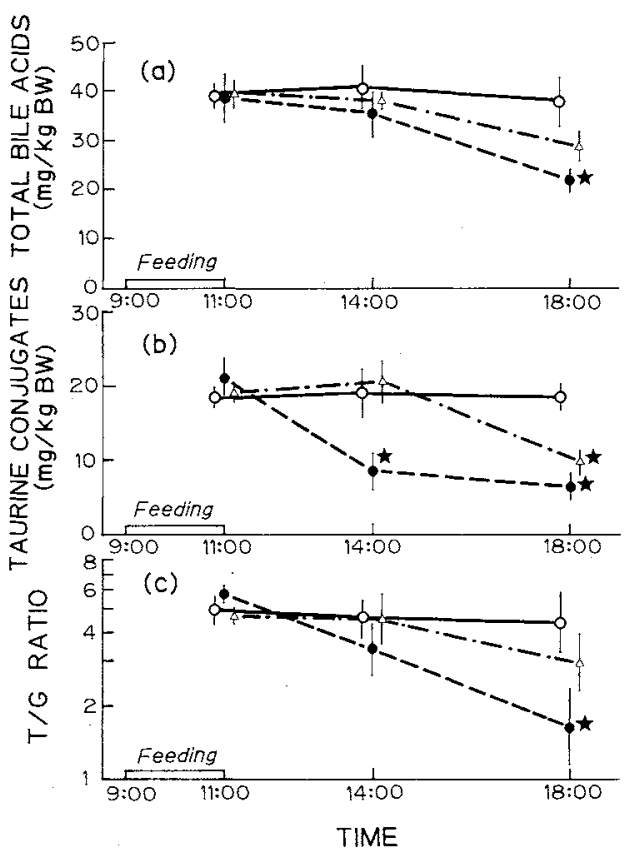

Fig. 1. Postprandial Changes in the Levels of (a) Total Bile Acids and (b) Taurine Conjugates in Rat Ileal Lumen and (c) in the $T / G$ Ratio.

Adult rats were fed for 4 weeks on the meal-feeding schedule described in the text. Each symbol and bar represents the mean $\pm \mathrm{SE}$ for 5 animals ( $\mathrm{O}-$, group $\mathrm{A}$; - - group B; $\triangle \triangle-$, group C). * Significantly different from the starting point $(11: 00)$ at $p<0.05$ on the basis of Student's $t$-test.

reflux, saponification at $120^{\circ} \mathrm{C}$ for $3 \mathrm{hr}$ with $10 \mathrm{~N} \mathrm{NaOH}$ and its subsequent extraction with chloroform-methanol $(2: 1)^{6)}$

Figure 1 depicts postprandial changes in the intraluminal levels of total bile acids (a) and taurine conjugates (b) and in the $T / G$ ratio (c) in the ileum of adult rats with alternation of diets during the period of 4 weeks on the meal-feeding schedule already described. The total bile acid content at 18:00 after ingesting the non-protein diet fell to half that at 11:00. Such was not the case with the group given the $40 \%$ casein diet at the stated feeding time in the morning; that is, there was no change in the intraluminal bile acid level in the ileum irrespective of fasting for a quarter of a day. A moderate decrease was observed in the control given the $20 \%$ casein diet at the same time period in the morning. These postprandial changes in the total bile acid levels were similar to those in the $\mathrm{T}$ levels as shown in Fig. 1(b).

On the other hand, the $G$ levels were in the average range of 3 to $5 \mathrm{mg} / \mathrm{kg}$ of body weight (BW), in which there were not significant differences among the three groups. For this reason, the postprandial variation of $\mathrm{T} / \mathrm{G}$ ratio faithfully reflected the fluctuation of $T$ levels as illustrated 
in Fig. 1(c). Interestingly, the $T / G$ ratio became less at 18:00 after ingesting the non-protein diet than after ingesting the high-protein diet, the control value being intermediate between them.

The intraluminal content in the ileum (strictly speaking, in the distal third of the small intestine) alone was used for bile acid measurements in this experiment. Bile which has been discharged into the duodenum does not stay there long. Chyme also seems to pass quickly through the duodenojejunum, so that the residues from its absorption come under observation in the ileum. ${ }^{71}$ In practice, the total bile acid content in the jejunal lumen was found to be far less than that in the ileal lumen (data not shown). This is the reason why the ileum was chosen for measuring the intraluminal bile acids. It is feasible that taurine conjugates are not much lost during the course of enterohepatic circulation and that their loss is compensated for by synthesis in the liver. Hepatic and/or plasma sulfur-containing amino acids and, more likely, hepatic glutathione (a major reservoir of cysteine within the body) ${ }^{8}$ presumably serve as taurine sources in the case of conjugation. Non-protein feeding leads to little increase in the hepatic glutathione content, while glutathione is maintained at a high level in the liver after ingesting a protein-rich diet. Taking into account these facts, a marked decrease in the $\mathrm{T} / \mathrm{G}$ ratio in rats after non-protein feeding may be explained by the lack of taurine supply due to a large reduction in the sulfur-containing amino acid pool. There remains still another possibility that $\mathrm{T}$ may have more promptly undergone either intestinal absorption or enterobacterial conversion than $\mathrm{G}$.

\section{References}

1) F. A. Wilson, Am. J. Physiol., 241, G83 (1981).

2) W. E. Elliot, in "Sterols and Bile Acids," ed. by H. Danielsson and J. Sjovall, Elsevier, Amsterdam, 1985, pp. 303-329.

3) W. G. Hardison and J. H. Proffit, Am. J. Physiol., 232, E75 (1977); J. A. Sturman, J. Nutr., 103, 1566 (1973).

4) Y. Yamanaka, K. Tsuji, T. Ichikawa, Y. Nakagawa and M. Kawamura, Sulfur Amino Acids, 8, 209 (1985); T. Ide and M. Horii, Brit. J. Nutr., 61, 545 (1985).

5) H. Von Ganshirt, F. W. Koss and K. Morianz, Artzneimit. Forsh., 10, 943 (1955).

6) S. M. Grundy, E. H. Ahrens Jr. and T. A. Miettinen, J. Lipid Res., 6, 397 (1965).

7) K. Iwami, M. Kitagawa and F. Ibuki, Nutr. Res., 10, 547 (1990).

8) N. Tateishi, T. Higashi, A. Naruse, K. Nakashima, H. Shiozaki and Y. Sakamoto, J. Nutr, 107, 51 (1977). 